\title{
Fiebre tifoidea: Emergencia, cúspide y declinación de una enfermedad infecciosa en Chile
}

\author{
Enrique Laval R. y Catterina Ferreccio R.
}

\section{Typhoid fever: rise, peak and fall of an infectious disease in Chile}

This article presents the history of typhoid fever in Chile since its definition as a clinical entity until our days. From this history is evident the long lasting confusion with typhus (rickettsial spotted fever) in Chile although the identity and characteristics of typhoid fever had been established in the first half of nineteenth century in Europe. This confusion could be explained because some clinical features are similar in both diseases (high fever and delirium) and because of its ocurrence in poor hygienic conditions. This misconception was resolved only during 1918 on occasion of a major typhus outbreak that allowed physicians to clearly diagnose this rickettsial disease. Once typhoid fever was recognized it was possible to describe its epidemiological pattern with high endemic incidence mainly in urban districts, with summer increases and epidemic cycles. In the contemporary history of typhoid fever it is remarkable a huge outbreak during 1976-1985, associated to abrupt socioeconomical and environment crisis, as well as an abrupt diminution of the disease in 1992, with a marked reduction that persists until now. This last phenomenon was the consequence of a cuasi-experimental public health intervention and sanitary education conducted in 1992 to avoid the cholera epidemic that was spreading in Perú, a neighboring country. We conclude that, although the hypothesis of environment contamination as the cornerstone in typhoid persistence was present since the recognition of the disease in 1894, it was faced efficiently only and perhaps in a definitely manner only almost 100 years later.

Key words: Typhoid fever, epidemiology, sanitary control.

Palabras claves: Fiebre tifoidea, epidemiología, control sanitario.

Emergencia de la fiebre tifoidea

como entidad nosológica:

Distinción con el tifus

exantemático

\section{Período de las fiebres tíficas}

Según Trousseau, es muy probable que se involucrara la fiebre tifoidea en las descripciones del Synochus putris (Cullen), Febris putrida (Stoll), Fiebre maligna nerviosa (Huxham), Fiebre mucosa (Roederer), Fiebre biliosa (Tirsot), Fiebre adinámica, ataxo-adinámica ${ }^{1}$.

Las clasificaciones, todas sintomáticas, publicadas por Pinel en 1798 en su "Nosografía de las fiebres esenciales", obligaron a la búsqueda de la fiebre tifoidea en cuatro o cinco capítulos diferentes, por lo general, mezclada con otras enfermedades infecciosas, sobre todo el tifus exantemático. A modo de ejemplo, en la "Historia de las Enfermedades en el Ejército Grande de Francia en las Campañas de Rusia en 1812 y de Alemania en 1813" de Kirckhoff, es posible deducir lo ya señalado, en los capítulos sobre "fiebre gástrica y de la fiebre mucosa con signos continuos y remitentes; de la fiebre lenta, del tifo y del sinoque", en este último con una evidente confusión con el tifus exantemático ${ }^{2,3}$.

Chile no constituyó una excepción en la confusión de la fiebre tifoidea con el tifus exantemático, como puede comprobarse en los textos de algunos antiguos historiadores de la medicina chilena ${ }^{4-6}$. La nebulosa entre ambas enfermedades continuó a pesar de los trabajos de Bretonneau, de Louis y, a que médicos tan distinguidos como Grajales, Cox y Blest, trabajaban en nuestros hospitales² $^{2}$ En 1856 llegó a Chile, entre otros médi- cos extranjeros, el italiano César Adami, el que para revalidar su título, leyó en la Facultad una memoria "Sobre las enfermedades más comunes en el ejército turco durante la Guerra de Oriente" en la que dijo "discusiones muy largas tuvieron luego entre los médicos de los varios ejércitos: sobre la identidad del tifo y de la fiebre tifoidea: para algunos la cuestión no está todavía resuelta. Por la práctica que he adquirido para mi, el uno y la otra son la misma enfermedad, con diferentes grados de gravedad y de duración, el tifo de una duración más corta y la fiebre tifoidea de una más larga"7.

Con ocasión de la epidemia de tifus exantemático a fines de 1864 y que continuó hasta 1866, el profesor Adolfo Valderrama comentó "las epidemias actuales son dos: las primeras constituyen las fiebres erup- 
tivas, entre las cuales llaman principalmente la atención las formas 'varioloides', denominadas vulgarmente con el nombre de 'peste' y la segunda una especie de fiebre tifoidea, todavía no bien caracteriza$d a$, pero que no tendría mucha semejanza con el 'typhus fever' o tifus epidémico de Inglaterra. Esta enfermedad designada como su congénere, con el nombre vulgar de 'chavalongo', es menos frecuente que las anteriores. Respecto del 'typhus fever o fiebre tifoidea epidémica', puede decirse que sus estragos se han limitado a un número relativamente muy inferior al de las viruelas, atacando como ellas, de preferencia a individuos menesterosos, que se entregan de ordinario al exceso de las bebidas alcohólicas o que se exponen a fuertes insolaciones, como sucede con los trabajadores del campo".

Concluye el profesor Valderrama: "la fiebre tifoidea, si es que así puede denominarse la fiebre que actualmente reina, es eminentemente contagiosa. He visto que todas las enfermeras de las salas han caido con ella y también las que las reemplazaron; cuatro Hermanas se han enfermado y las cuatro de fiebre tifoidea. La epidemia es, por lo tanto, muy seria y no creo que deba ocultársele al Gobierno como para que tome algunas medidas, aunque nuestros gobiernos jamás han hecho nada por la salubridad pública"8. Sin lugar a dudas, la epidemia fue de tifus exantemático y causó la muerte del doctor Lorenzo Sazie el 30 de noviembre de 1865 .

Lo más notable sucedió algunos años más tarde, cuando el alumno del $6^{\circ}$ año, don Florencio Middleton, presentó en 1867 a la Facultad de Medicina una memoria sobre typhus fever relativa a lo sucedido entre los años 1864 y 1866, aunque el tema que la Facultad de Medicina había acordado para ese certamen era "Fiebre tifoidea en Chile, sus causas, desarrollo, tratamiento, anatomía patológica y sus diferencias respecto de la de otros países". Sin embargo, Middleton afirmó: "que la existencia del typhus fever, al cual se le da impropiamente el nombre de fiebre tifoidea, su extensión a toda la República, la mortalidad que alcanza a una cuarta parte de los atacados prácticamente en las épocas de mayor gravedad, etc., hacen cada día más importante el estudio de esta enfermedad. Mi revisión comprende el año 1864 (desde agosto), 1865 y 1866, con la interrupción de los últimos meses de 1865, a consecuencia de haber contraido yo mismo el tifus, durante el estudio hecho expresamente para la presentación de esta memoria. Llamo la atención -continúa Florencio Middleton- que inclui dos observaciones de dotinentería o tifus abdominal que pueden dar alguna idea de las diferencias capitales que esta afección presenta respecto de la que es objeto el presente trabajo, en lo que hace a las vías digestivas y sus dependencias principalmente".

El informe final de esta memoria realizado por los doctores Adolfo Valderrama y Carlos Leiva, otorgó el premio a lo que fue considerado... ¡como el mejor trabajo sobre fiebre tifoidea! ${ }^{9}$.

En el año 1868 el doctor Germán Schneider, en "algunas observaciones sobre diphteritis, typhus, viruela y reflexiones sobre nuestras instituciones médicas", manifiesta que "poco más o menos he visto en los últimos seis años unos quinientos enfermos de tifus, naturalmente entre ellos muchos casos leves, pero también un buen número de casos más graves. En mis apuntes he notado los primeros, los leves, como fiebre tifoidea y los segundos como tifus". O sea, lo contrario de lo expuesto por el doctor César Adami ${ }^{10}$.

El profesor Wenceslao Díaz Gallego aseguraba en 1875, que la fiebre tifoidea se presenta en Chile "con los mismos caracteres que en Europa, sin que nunca se haya podido comprobar su carácter contagioso ni haya reinado epidémicamente". Además, afirma perentoriamente que el tifus exantemático sólo apareció en Chile después de $1864^{11}$.

En un curioso librito escrito en 1881, por el doctor Joaquín Zelaya, con el título de "Estudio práctico sobre la fiebre tifoidea de Chile", expone que "la tifoidea es la fiebre más grave que existe en Chile y forma un tipo regular y característico, advirtiendo al Cuerpo Médico que este estudio es el resultado de mi práctica; mi manera de ver y apreciar esta enfermedad, después de haberla observado en casi todos los pueblos de Chile". Agrega que "en muchas ocasiones aparece la tifoidea epidémica bajo una forma más o menos seria, precediendo a las viruelas $u$ otras constituciones médicas desfavorables. Puede decirse que es endémica, en verano sobre todo, en

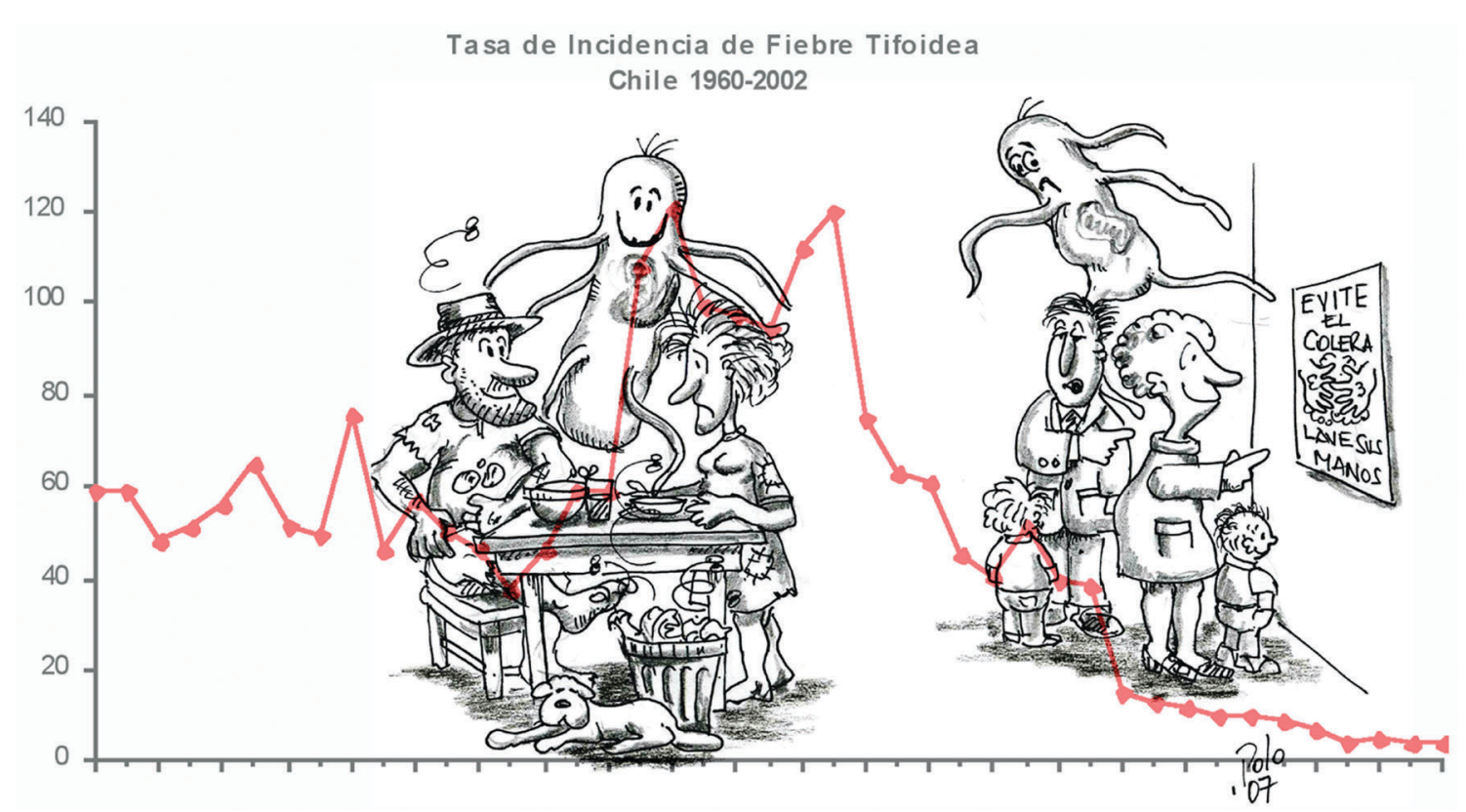


pueblos situados en lechos de ríos circunvalados de vegas, depósitos de aguas estancadas y en los puertos cuyas riberas son sucias. Sin embargo, es muy satisfactorio que la forma epidémica no es frecuente en el país. Sólo una vez hace 16 años lo fue en Santiago y en otros pueblos, pero nunca ha tenido las proporciones de la viruela y escarlatina de otras épocas"12.

Es importante recordar y destacar, como excepcional, en aquella época de confusión diagnóstica, la memoria del doctor Moisés Amaral en el año 1886, para optar al grado de Licenciado en la Facultad de Medicina y Farmacia, intitulada "Consideraciones clínicas sobre la fiebre tifoidea en Chile". Este basado en sus propias experiencias, describe prolijamente las características clínicas de la enfermedad, resaltando los principales síntomas y signos con relación al diagnóstico y pronóstico. Interesante y pintoresco es cuando menciona la ausencia de alcantarillado, al relatar "los casos engendrados por la infección miasmática" y en un arranque de rabia e impotencia exclama: "inunca se dirá lo bastante respecto de este incalificable procedimiento de abandonar en la calle pública el putrílago infecto de las acequias! La autoridad prohíbe la venta de sustancias venenosas en las boticas, siempre que un facultativo no las prescriba y sin embargo, permite que al frente de chas casas se depositen sustancias tan deletéreas, miasmas tan venenosos que atacan seriamente nuestra salud, cuando no terminan con nuestra vida"13.

En 1902, el doctor Eduardo Degeyter Carmona, médico de "ciudad y vacuna" en Rancagua desde 1897, publica una epidemia de fiebre tifoidea producida en dicha ciudad y pueblos cercanos, en el verano de 1901, informando que "la fiebre tifoidea es endémica en Rancagua, lo que no es raro, pues el agua del río Cachapoal que sirve de bebida a sus habitantes, no puede ser peor; a veces su olor es tan putrefacto, que no adivino por qué no se ha hecho epidémica". Relata las vicisitudes de 73 enfermos internados en el hospital de la ciudad, de los que fallecieron cuatro, declarándose partidario fervoroso del tratamiento con agua clorofórmica adicionada con magnesia fluida, benzoato de soda y bromuro de sodio, siguiendo las indicaciones de los médicos alemanes Werner y Stepp, por lo que "me creo en el deber de manifestar por la prensa médica los buenos resultados obtenidos con este sencillo y racional tratamiento" ${ }^{14}$. Es muy probable que varios de los enfermos descritos por el doctor Degeyter, pudieran haber correspondido a tifus exantemático (comienzo brusco, presencia de exantema petequial, normalización de la temperatura al cabo de 8 a 10 días).

\section{Separación de la fiebre tifoidea del tifus exantemático}

Recordemos que sólo en 1894, el Consejo Superior de Higiene presentó al Gobierno un proyecto de nomenclatura de las causas de muerte y por vez primera, en forma oficial, hace la separación de la fiebre tifoidea del tifus exantemático ${ }^{15}$. Finalmente, a partir de la gran epidemia de tifus exantemático en 1918, se comenzó a diferenciarlas con precisión, demostrando en forma irrefutable el doctor Arturo Atria Osorio que el tifus exantemático había existido desde siempre en Chile, como una entidad clínica propia y característica. Además en el año 1919, se empezó a efectuar en el país la reacción de Weil-Felix, contribuyendo a la confirmación del diagnóstico a través del laboratorio ${ }^{16,17}$.

\section{Inicio de la historia de la fiebre tifoidea en Chile}

En el año 1919, en un informe de la Dirección de Estadística sobre "las enfermedades denunciables" en el país (incluida Tacna), referido a enfermos hospitalizados, la morbimortalidad de la fiebre tifoidea superó a la difteria, tifus exantemático, viruela, escarlatina y peste bubónica. Así, de 2.120 enfermos, fallecieron 434 (20,7\%), por lo que el doctor Ricardo Dávila Boza, Director del instituto de Higiene, comunicó al Presidente del Consejo Superior de Higiene "que la fiebre tifoidea ha tenido en la temporada de verano del presente año un desarrollo superior al de igual época del año pasado de más de $50 \%$ y de cerca del $100 \%$ en relación al mes de febrero. Felizmente en el mes de marzo ha habido alguna disminución" 18 .

En los años siguientes, la fiebre tifoidea continuaría en Chile con las características de una enfermedad infecciosa endémica, con cifras de morbilidad que varían entre 50 y 80 por 100.000 habitantes, presentando de vez en cuando, episodios catalogados de "epidemia", que superarían los valores máximos de la endemia, como ha sido relatado en varias publicaciones. Algunos referidos a casi todo el país y otros a focos localizados en distintas provincias y ciudades; limitados en otras ocasiones a instituciones cerradas, como establecimientos educacionales y $\operatorname{asilos}^{19-26}$.

La cifra de mortalidad, cuyo registro oficial más antiguo corresponde al año 1903, fue de 46,9 por 100.000 habitantes, aumentando a más del doble entre 1906 y 1909 , aunque es posible que sean valores abultados debido a la confusión con el tifus exantemático. A partir de 1932 la mortalidad por fiebre tifoidea bajó a un dígito, con un alza en algunos años de la década de 1940. A partir de 1950, se observó un descenso importante, que se explica por la introducción del cloranfenicol como tratamiento específico de la enfermedad ${ }^{23,25}$.

Viel relata que "la onda epidémica de la fiebre tifoidea que comenzó en 1946, alcanzando hasta 1948, fue un fenómeno circunscrito a la ciudad de Santiago, siendo la tasa de morbilidad más alta la de 1946 $(102,5 / 100.000)^{21}$.

En el período comprendido entre el $1^{\circ}$ de octubre de 1956 y el 30 de junio de 1957, se produjeron en la provincia de Santiago 2.812 casos y 32 defunciones, lo que representó tasas de morbilidad y mortalidad por 100.000 habitantes de hasta 130,3 y 1,6 , respectivamente; lo que según Borgoño "de acuerdo con los estudios estadísticos, el número de casos notificados entre noviembre de 1956 y mayo de 1957, fue superior al de casos esperados, lo que significó un alza epidémica debidamente sustentada". Una de las causas de esta situación, podría haber sido aparentemente, el incremento del fagotipo B3 y la aparición del C en Salmonella typhi, lo que estaría en relación con nuevos portadores situados en posiciones estratégicas, que habrían contribuido a la manutención de la endemia y a la producción del brote epidémico 1956-1957. Respecto a este suceso, advierte Borgoño, "que si el conjunto de acciones destinadas a solucionar los principales problemas de saneamiento ambien- 
tal, a través de políticas de Gobierno, asi como el control de alimentos, de moscas, prohibición de riego de verduras y hortalizas con aguas servidas, etc, no son llevadas a cabo, es difícil no pronosticar que en los próximos años la fiebre tifoidea y otras infecciones entéricas, continuarán teniendo elevados indices de morbi-mortalidad" 24 .

\section{Cúspide y ocaso de la fiebre tifoidea en Chile}

Desde los años 50 la fiebre tifoidea se presentó en Chile en forma endémica, con tasas de incidencia anuales por 100.000 habitantes (tasa) en torno a 60 y con aumentos y disminuciones cíclicas llegando a tasas de hasta 80 . Por sobre estos ciclos, de 4 años aproximadamente, la fiebre tifoidea tenía una leve tendencia secular descendente, registrándose el nivel más bajo en Chile en 1973 con 3.688 casos y tasa de 37,3. Sin embargo, sólo cuatro años más tarde se desencadenó una de las mayores epidemias de fiebre tifoidea descritas en el país; entre 1976 y 1977 los casos anuales subieron de 6.180 a 11.533 con un máximo de 13.144 casos en 1978. Las tasas medias duplicaron la media histórica llegando a 121 a nivel nacional con la tasa máxima regional de 177 en la Región Metropolitana. Las muertes anuales por fiebre tifoidea en el país subieron de 56 a 120 entre 1973 y 1977, pero la letalidad continuó disminuyendo. La fiebre tifoidea se mantuvo en este nivel epidémico por cerca de una década lo que le valió el apelativo de "hiperendemia" (Figura 1) ${ }^{27}$.

La epidemia afectó a toda la población pero principalmente a las personas de menor nivel socioeconómico, en Santiago por ejemplo, la mayor tasa fue en San Miguel y la más baja en Las Condes, con 266,2 y 45,6 respectivamente. La edad media de los casos fue 15,5 años, con las tasas más altas entre los 5 y 25 años $^{27}$.

El mayor riesgo en Santiago con respecto al país ocurría desde antes de la "hiperendemia" y principalmente en los meses de verano, cuando los casos en Santiago duplicaban al resto de Chile, mientras en invierno los igualaban ${ }^{27}$. La estacionalidad de la fiebre tifoidea se mantuvo durante todo el período epidémico.
En Santiago, a partir de 1986 empezó a decrecer la epidemia bajando de 7.000 a 2.000 casos anuales en 1990. En base a su comportamiento histórico se esperaba que la fiebre tifoidea se mantuviera en torno a los 2.000 casos anuales, con alzas de hasta $4.000 \mathrm{y}$ bajas hasta $1.000 \operatorname{casos}^{28}$. Sin embargo, en el año 1992 presentó una baja histórica en Chile, pero principalmente en Santiago, donde llegó a sólo 300 casos anuales. Con esta caída terminan los ciclos de la fiebre tifoidea, siendo reemplazados por un descenso sostenido que aún sigue por más de 14 años. En 2006 rompió un nuevo récord bajando la cuenta anual de casos en Santiago a dos dígitos 78 casos (tasa 1,2), 100 veces menor que a mediados de los 80 (Figura 1).

Aunque se ha descrito y debatido bastante el comportamiento de la fiebre tifoidea en Chile y en particular en Santiago, la historia natural de la epidemia es más clara hoy cuando se pueden evaluar los fenómenos sociales que la determinaron.

\section{¿Por qué ocurrió la epidemia de fiebre tifoidea de 1977 a 1985?}

El aumento explosivo de la fiebre tifoidea a fines de los años 70, que duraría cerca de ocho años, se podría explicar por el efecto sinérgico de la contaminación ambiental, la caída del control sanitario de los alimentos y el deterioro de las condiciones de vida de la población.

Respecto de este último punto, citamos a Castañeda: "... Los cambios más bruscos en empleo y remuneraciones reales en Chile ocurrieron en la década de 1970. Las caídas más fuertes en empleo fueron en 1974 $(-3,7), 1975(-2,1)$ y $1982(-9,1)$. Las caidas más fuertes en las remuneraciones reales, por su parte, ocurrieron en 1972 (-19,9); $1973(-47,4)$ y 1983 (-10,7). La caida de los salarios en 1973 llevó a éstos a su nivel más bajo (a la mitad del nivel de 1970) siendo incluso su nivel menor al que ellos tenían en 1960...". En este período también hubo una importante disminución del gasto público $^{29}$.

En estos años de recesión se acentuó la migración del campo a las ciudades, lo que implica un influjo de personas susceptibles a la fiebre tifoidea, que llegan a un ambiente en condiciones de mayor transmisión, pro- ducto del empobrecimiento (hacinamiento, falta de higiene) y de la fuerte restricción al control de alimentos ${ }^{30}$.

Es interesante destacar que en estos años de disminución del gasto público, se mantuvo y aumentó la cobertura de agua potable y alcantarillado en todas las áreas urbanas ${ }^{29}$. Esta mejoría de la cobertura del saneamiento básico, que impactó positivamente en la mortalidad infantil, tuvo un efecto paradójico en Santiago. La principal fuente de contaminación ambiental en la capital de Chile era el alcantarillado que descargaba directamente y sin tratamiento, en el río Mapocho. Un efecto negativo del aumento de cobertura de alcantarillado es que se incrementa el nivel de microorganismos patógenos en las aguas del río, entre ellos $S$. typhi ${ }^{31}$. Estas aguas se usaban para el riego de los alrededores de Santiago y a través de la contaminación de las hortalizas cultivadas en estas tierras se distribuía la fiebre tifoidea a las clases de nivel socioeconómico alto, quienes no estuvieron libres de la enfermedad en esos años.

Es esta amplificación de la transmisibilidad de S. typhi a través de la contaminación ambiental de las hortalizas lo que explica el exceso relativo de la fiebre tifoidea en la ciudad de Santiago respecto del resto de Chile $^{28}$.

\section{El ocaso de la fiebre tifoidea}

La respuesta a la epidemia en términos de campañas de educación sanitaria, prohibición de uso de aguas servidas, vacunación anti-tífica (aunque con eficacia moderada de 60-75\%, llegaron a cubrir a 400.000 escolares en Santiago) y en parte el agotamiento de los susceptibles dieron cuenta de la declinación paulatina de la epidemia entre 1986-91.

Esta lenta declinación fue interrumpida por una brusca aceleración que en sólo un año, de 1991 a 1992, redujo en 81,2\% la incidencia de fiebre tifoidea. Esta caída estuvo asociada a una intervención de salud pública -de carácter cuasi-experimental- para interrumpir la transmisión del Vibrio cholerae en Chile. Esta intervención, que se inició en marzo del año 91 en repuesta a la epidemia de cólera de Perú, consistió en la prohibición absoluta del riego con aguas servidas en todo el país, prohibición absoluta del 
expendio para consumo en lugares públicos de verduras crudas, cloración de canales de riesgo y control estricto del cloro residual del agua potable, campañas de comunicación masiva de higiene y lavado de manos, y refuerzo de controles sanitarios en las fronteras.

Esas intervenciones evitaron el brote estacional del verano de 1992 y dieron comienzo a un circuito virtuoso que llevó en la práctica a la desaparición de la fiebre tifoidea de nuestros hospitales (Figura 1). En ese momento ocurrió también la concatenación de fenómenos pero esta vez en el sentido contrario. En efecto, a la toma de decisiones de la autoridad para evitar la transmisión ambiental de patógenos entéricos se sumó la mejoría de las condiciones de vida de la población que empezó a exigir alimentos higiénicos. Se iniciaron obras de ingeniería que mejoraron las condiciones ambientales y prácticamente desapareció el cultivo de hortalizas en las zonas aledañas a Santiago.

\section{Incluso la estacionalidad desaparece}

La fuerte estacionalidad de la fiebre tifoidea es propia de una infección con un importante componente ambiental en su mecanismo de transmisión. En la medida que el componente ambiental se controló, la fiebre tifoidea en la RM prácticamente perdió su estacionalidad; actualmente ocurren casos a lo largo de todo el año, probablemente asociados a portadores crónicos de $S$ typhi. Por ello, las medidas de control hoy, se deben centrar en la higiene y control de los alimentos y en la educación sanitaria y control de los manipuladores de alimentos. Este mecanismo siempre estuvo presente, pero ampliamente sobrepasado por la amplificación de la epidemia que produce el componente ambiental.

El apasionado debate sobre las causas de la alta endemia de fiebre tifoidea en Chile donde unos preferían atribuirlo a la provisión de agua potable de mala calidad ${ }^{32}$, otros a los portadores crónicos y en particular a los manipuladores de alimentos ${ }^{30}$, otros se declaraban ignorantes ${ }^{25}$ y otros planteaban la acción sinérgica de varios de ellos incluido el deterioro de las condiciones de vida $^{27}$ podríamos concluir diciendo, todos tenían razón, sólo había que actuar.

\section{Resumen}

En este artículo se presenta la historia de la fiebre tifoidea (FT) en Chile desde su reconocimiento como entidad nosológica hasta su situación actual. De la historia destaca la confusión que hubo en Chile durante muchos años entre esta enfermedad y el tifus exantemático a pesar de que la fiebre tifoidea ya había sido individualizada y caracterizada en la primera mitad del siglo XIX en Europa. Esto se podría explicar porque tenían ciertas manifestaciones clínicas similares (fiebre alta y delirio) y por ocurrir en condiciones de déficit ambiental. Esta confusión se soluciona recién en 1918 en relación con la gran epidemia de tifus exantemático que permite a los clínicos identificarlo con toda claridad. Una vez distinguida la FT fue posible describir su comportamiento, caracterizado por un alto nivel de endemia, de preferencia en zonas urbanas con alzas estivales y ciclos epidémicos. De la historia contemporánea de la FT resalta la Gran Epidemia de 1976-1985, asociada al brusco deterioro socioeconómico y ambiental, y la igualmente abrupta caída de la enfermedad en 1992, reducción que persiste hasta nuestros días. Este último fenómeno, fue el resultado del carácter cuasi-experimental de las intervenciones de salud pública y educación sanitaria realizadas en 1992 para evitar la epidemia de cólera que estaba extendiéndose en el Perú. Concluimos que, si bien la hipótesis de la contaminación ambiental como el factor clave en la persistencia de la FT estuvo presente desde el reconocimiento de la enfermedad en 1894, sólo se abordó de manera eficaz y tal vez definitiva casi 100 años más tarde.

\section{Referencias}

1.- Pedro-Pons A, Farreras V P, Surós F J. Enfermedades Infecciosas. Tomo VI. p. 212-3. Salvat Ed. Barcelona. España. 1952.

2.- Laval R E. Bretonneau y Louis: diferenciación y caracterización de la fiebre tifoidea. Rev Chil Infect 2005; 22: 102-5.

3.- Kirckhoff J R E. Historia de las enfermedades observadas en el Ejército Grande de Francia en las campañas de Rusia en 1812 y de Alemania en 1813. p. 119-66. Impta. de Colo-Colo. Santiago. Chile. 1838.
4.- Salas O E. Historia de la Medicina en Chile. p. 107-9 y 230-86. Impta. Vicuña Mackenna. Santiago. Chile. 1894.

5.- Grossi J. Reseña del Progreso Médico en Chile. p. 378. Impta. de la Opinión. Valparaíso. Chile. 1895.

6.- Ferrer P L. Historia General de la Medicina en Chile. p. 257. Impta. Talca. Chile. 1904.

7.- Adami C. Sobre las enfermedades más corrientes en el ejército turco durante la Guerra de Oriente. AUCH 1858; 15: 169.

8.- Documentos sobre el actual estado epidémico de Santiago. AUCH 1864; 24: 872-80.

9.- Middleton, F. Memoria sobre la epidemia de typhus-fever presentada a la Facultad de Medicina, por el alumno de $6^{\circ}$ año, don Florencio Middleton, premiada en el certamen de 1867. AUCH 1871; 44: 563-81.

10.- Schneider G. Algunas observaciones sobre diphteritis, typhus, viruela y reflexiones sobre nuestras instituciones médicas. AUCH 1868; 30: 563-81.

11.- Diaz G W. Enfermedades reinantes en Chile. Capítulo III: De las enfermedades epidémicas. AUCH. 1875, 86-114.

12.- Zelaya, J. Estudio práctico sobre la fiebre tifoidea de Chile. Impta. de la Reforma. Serena. Julio de 1881.

13. - Amaral M. Fiebre tifoidea. Consideraciones clínicas. Rev Méd Chile 1886; 15: 242-56.

14.- Degeyter C E. Epidemia de fiebre tifoidea en Rancagua: su tratamiento con agua clorofórmica. Rev Méd Chile 1902, 30: 183-93.

15. - Laval M E. Desarrollo histórico de la fiebre tifoidea en Chile. (apuntes de clases inéditos). 1957.

16.- Atria O A. Sobre tifus exantemático. p. 3.-64. Impta. Franco Chilena. Santiago. Chile. 1919.

17.- Laval M E. Diagnóstico del tifus exantemático por la Reacción de Weil-Felix. Tesis para optar al grado de Licenciado de la Facultad de Medicina. Impta. Universitaria. Santiago de Chile. 1919.

18. - Dávila B R. Fiebre Tifoidea en el verano de 1918. Rev Chil Higiene 1919; 25: 55-8.

19. - Molina G. Estudio epidemiológico de la fiebre tifoidea en Santiago, con especial referencia al brote epidémico estacional 1939-1940. Rev Chil Higiene y Med Prev 1940, 3: 169-97.

20.- Donoso J. Anotaciones sobre epidemiología de la fiebre tifoidea en Chile. Rev Méd Chile 1942; 70: 251-60.

21.- Viel V B, Ebensperger I. Estudio epidemiológico de la fiebre tifoidea en la provincia de Santiago. Rev Chil Higiene y Med Prev 1950; 13: 103-25.

22.- Romero H, Reyes E, Amarales J, 
Vildósola J. Aporte a la epidemiología de la fiebre tifoidea. Evolución y estado actual de la endemia. Rev Chil Higiene y Med Prev 1951; 13: 51-64.

23.- Borgoño J M, Latorre M. Estado actual de la epidemiología de la fiebre tifoidea en Santiago. Rev Chil Higiene y Med Prev 1953; 15: 53-64.

24.- Borgoño J M. Nuevos aportes a la epidemiología de la fiebre tifoidea en la provincia de Santiago, con especial referencia al brote epidémico estacional 1956-1957. Rev Méd Chile 1958; 86: 413-9.

25.- Quesney F. Algunas consideraciones sobre epidemiología de la fiebre tifoidea. Bol Esc
Med PUC 1983; 30: 113-9.

26.- Caballero J. Investigación del origen de la epidemia de tifoidea en el Asilo Baquedano. Rev Méd Chile 1939; 67: 1187-97.

27.- Ferreccio C. Estudio de la Salmonella typhi en cursos de agua de la ciudad de Santiago. Tesis de Grado. Magíster en Salud Pública. Escuela de Salud Pública. Universidad de Chile. Santiago de Chile 1983.

28.- Ferreccio C, Ortiz E, Pino M, Zamora D. Asociaciones epidemiológicas entre contaminación del agua y daño en salud en la Región Metropolitana de Chile. Monografía. Organización Panamericana de la Salud. Santiago Chile 1993
29.- Castañeda T. Contexto socioeconómico y causas del descenso de la mortalidad infantil en Chile. Centro de Estudios Públicos 1984.

30.- Medina E, Irarrázaval M. Fiebre tifoidea en Chile: consideraciones epidemiológicas. Rev Méd Chile 1983; 111: 609-15.

31.- Sears S, Ferreccio C, Levine M M Sensitivity of Moore swabs for isolating Salmonella typhi from irrigation water in Santiago, Chile. J Infect Dis 1984; 149: 640-2.

32.- Cabello F, Agüero M E, Fernández M. Epidemia de fiebre tifoidea en Chile: aspectos ecológicos y microbiológicos. Rev Méd Chile 1984; 112: 826-8. 